\title{
Measuring skin temperature before, during and after exercise: a comparison of thermocouples and infrared thermography
}

\begin{abstract}
Alex de Andrade Fernandes $1,2,5$, Paulo Roberto dos Santos Amorim ${ }^{1}$, Ciro José Brito ${ }^{3}$, Anselmo Gomes de Moura ${ }^{1}$, Danilo Gomes Moreira ${ }^{1}$, Carlos Magno Amaral Costa ${ }^{1}$, Manuel Sillero-Quintana ${ }^{4}$ and João Carlos Bouzas Marins ${ }^{1}$
\end{abstract}

\footnotetext{
${ }^{1}$ Department of Physical Education, Human Performance Laboratory, Federal University of Viçosa, Viçosa, Brazil

${ }^{2}$ Federal Institute for Education, Sciences and Technology of Minas Gerais, Bambuí, Brazil

${ }^{3}$ Department of Physical Education, Federal University of Sergipe, Aracaju, Brazil

${ }^{4}$ Faculty of Physical Activity and Sport Sciences, Polytechnic University of Madrid, Madrid, Spain

E-mail: alex.andrade@ifmg.edu.br
}

Received 1 July 2013, revised 28 November 2013

Accepted for publication 3 December 2013

Published 7 January 2014

\begin{abstract}
Measuring skin temperature $\left(T_{\mathrm{SK}}\right)$ provides important information about the complex thermal control system and could be interesting when carrying out studies about thermoregulation. The most common method to record $T_{\mathrm{SK}}$ involves thermocouples at specific locations; however, the use of infrared thermal imaging (IRT) has increased. The two methods use different physical processes to measure $T_{\mathrm{SK}}$, and each has advantages and disadvantages. Therefore, the objective of this study was to compare the mean skin temperature $\left(M T_{\mathrm{SK}}\right)$ measurements using thermocouples and IRT in three different situations: pre-exercise, exercise and post-exercise. Analysis of the residual scores in Bland-Altman plots showed poor agreement between the $M T_{\mathrm{SK}}$ obtained using thermocouples and those using IRT. The averaged error was $-0.75^{\circ} \mathrm{C}$ during pre-exercise, $1.22^{\circ} \mathrm{C}$ during exercise and $-1.16^{\circ} \mathrm{C}$ during post-exercise, and the reliability between the methods was low in the pre(ICC $=0.75[0.12$ to 0.93$]$ ), during ( $\mathrm{ICC}=0.49[-0.80$ to 0.85$])$ and post-exercise (ICC $=0.35[-1.22$ to 0.81$]$ conditions. Thus, there is poor
\end{abstract}

5 Address for correspondence: Department of Physical Education, Federal University of Viçosa, Avenida PH Rolfs s/n-Centro. Viçosa-Minas Gerais, 36571-000, Brazil. 
correlation between the values of $M T_{\mathrm{SK}}$ measured by thermocouples and IRT pre-exercise, exercise and post-exercise, and low reliability between the two forms of measurement.

Keywords: skin temperature, thermocouples, infrared thermal imaging, exercise

(Some figures may appear in colour only in the online journal)

\section{Introduction}

The skin is an essential organ for maintaining the core temperature $\left(T_{\mathrm{C}}\right)$ within the normal range of 36.1 to $37.8{ }^{\circ} \mathrm{C}$ in order to preserve the vital functions of the body (Campbell 2011). Thermal receptors in the skin identify the environmental conditions and generate adaptive physiological responses including peripheral vasoconstriction for the reduced skin temperature $\left(T_{\mathrm{SK}}\right)$ induced by low-temperature environments, and peripheral vasodilatation for the increased $T_{\mathrm{SK}}$ in response to elevated temperatures (Charkoudian 2010, Johnson and Kellogg 2010).

Exercise induces the transformation of chemical energy into kinetic energy and thermal energy, thus generating an increase in heat production. Heat production increases body temperature, especially in the active muscles, causing an inversion of the temperature gradient between muscles and arterial blood. Blood from the deeper regions of the body is cooled in the skin by evaporation contributing to the control of $T_{\mathrm{C}}$ (Shibasaki et al 2006, Johnson 2010, Johnson and Kellogg 2010, Charkoudian 2010).

Measuring $T_{\mathrm{SK}}$ provides information about the complex thermal control system and it seems interesting for carrying out studies about thermoregulation. Various methods have been used for this purpose including infrared thermal imaging (IRT), mercury, chemical thermometers and different types of thermocouples (Davie and Amoore 2010, Smith et al 2010). Thermocouples are the most commonly employed method for measuring $T_{\mathrm{SK}}$. Their operation is based on the phenomenon known as the Seebeck effect (Childs 2001), which occurs when there is a potential difference (voltage) between two conductor (or semiconductor) materials at different temperatures (Childs 2001).

The main advantages of using thermocouples include the low cost of the consumables, high accuracy, sensitivity and reproducibility, short response time and high range of temperature measurements. Furthermore, thermocouples can be used to measure $T_{\mathrm{SK}}$ during exercise and, in some models, without any cables, thus reducing the time required to set up the equipment and providing more mobility to the subject (Childs 2001, Smith et al 2010, Hasselberg et al 2011). The disadvantages of thermocouples include the following: small measurement area of a few $\mathrm{cm}^{2}$ (Smith et al 2010), relatively high cost of some equipment (Smith et al 2010), differences in the $T_{\mathrm{SK}}$ based on the technique for attaching the thermocouple (Tyler 2011), and complications due to heat loss through convection and evaporation in the region where the thermocouple is fixed (Tyler 2011, Buono et al 2007). Moreover, for long duration exercise, sweat and body movements can cause the thermocouple to detach (Buono et al 2007) and cause interference during movements when cables are used (Smith et al 2010).

In the past few years, IRT has gained prominence in $T_{\mathrm{SK}}$ measurements (Ring and Ammer 2012, Fernandes et al 2012). IRT is based on several physical principles, including the laws of Max Planck, Wilhelm Wien and Stefan-Boltzmann (Jones 1998, Bouzida et al 2009, Lahiri et al 2012). Objects at a temperature above absolute zero $\left(0 \mathrm{~K}\right.$ or $\left.-273.16^{\circ} \mathrm{C}\right)$ emit infrared radiation due to the thermal motion of atoms and molecules; thus, for larger agitations, the 
temperature of the object increases, and the object releases more infrared radiation (Jones 1998, Kennedy et al 2009, Lahiri et al 2012). Infrared cameras have special lenses that concentrate the thermal energy on an infrared detector consisting of thousands of infrared sensors (pixels) that transform infrared radiation into an electrical signal. The electrical signal is processed to show a thermal image on a display or video monitor and to calculate the temperature of each pixel (Jones 1998, Kennedy et al 2009, Lahiri et al 2012).

The main advantages of using IRT to measure $T_{\mathrm{SK}}$ include the following: the noninvasiveness of the technique, which does not require physical contact with the subject (Fernandes et al 2012, Vargas et al 2009); the capability of monitoring $T_{\mathrm{SK}}$ by focusing on a specific body region of interest (ROI) (local analysis) or on the entire body (global analysis) (Fernandes et al 2012, Vargas et al 2009, Bouzida et al 2009); the freedom of movement during exercise; the lack of interference in the processes of heat loss through radiation, convection and evaporation; the high sensitivity, accuracy and reproducibility; and the possibility of recording video with certain camera models (Fernandes et al 2012, Vargas et al 2009, van den Heuvel et al 2003, Ahmadi et al 2011, Zaproudina et al 2008, McCoy et al 2011). The disadvantages include the relatively high cost of the cameras with video or high definition, the lower precision of the equipment with simpler technology, the need to interrupt the exercise for recording when the camera has not recorded video, the need for specific training to control different factors that could affect the measurements, and the long time required to capture the data from the thermograms because the analysis software provided by the manufacturers is not specific to humans.

As described above, the IRT and thermocouples are based on different physical processes for measuring $T_{\mathrm{SK}}$, and each has advantages and disadvantages. Due to the current widespread use of thermocouples and the recent increase in the use of IRT, it seems important to check whether the $T_{\mathrm{SK}}$ values obtained using thermocouples are consistent with those measured using IRT. The objective of this study was to compare the mean skin temperature $\left(M T_{\mathrm{SK}}\right)$ measured using thermocouples to those measured using IRT and to check whether there is agreement between the two methods at different times (pre-, during and post-exercise).

\section{Methods}

\section{Participants}

After disclosing the dynamics and objectives of the study via e-mail to the physical education students on the UFV campus, 12 people volunteered (age $=22.4 \pm 3.3$ years, height: $177.0 \pm 0.8 \mathrm{~cm}$, body fat percentage: $10.3 \pm 3.0 \%$, body surface area: $1.92 \pm 0.09 \mathrm{~m}^{2}$ and $\dot{V} \mathrm{O}_{2 \max }: 48.7 \pm 4.9 \mathrm{ml} \mathrm{kg} \mathrm{min}^{-1}$ ). They were previously informed about the procedures for all stages of the investigation and signed the informed consent forms. This study was approved by the local Ethics Committee on Human Research (No 134, 2011).

\section{Research model}

This study consisted of a randomized model. The $T_{\mathrm{SK}}$ of half of the subjects was first measured using thermocouples and later using IRT, while the second half was first evaluated using IRT and later using thermocouples. The interval between measurements was more than two days and less than seven.

\section{Pre-experimental season}

Because external and internal factors can interfere with $T_{\mathrm{SK}}$ recordings, the following characteristics were used as exclusion criteria: smoking; history of kidney problems; injury; 
osteomyoarticular problems or symptoms in the last two months; skin burns, regardless of how the body areas were evaluated; symptoms of pain in any body region; sleep disorders; fever in the last seven days; physical therapy treatments; use of dermatological creams, ointments or lotions; and usage of medications, such as antipyretics or diuretics, or any dietary supplement that could potentially interfere with water homeostasis and body temperature in the last two weeks.

All subjects were considered apparently healthy according to the physical activity readiness questionnaire (Chisholm et al 1975) and showed low coronary risk according to the proposal of the Michigan Heart Association (McArdle et al 2001). The subjects were considered physically active subjects according to the criteria of the American College of Sports Medicine (ACSM) (Garber et al 2011); the subjects performed regular physical training sessions at least three times a week for four months.

In this phase of the study, the body mass in grams (Filizola ${ }^{\circledR}$, Star 300/4) and the height in centimetres (American Medical ${ }^{\circledR}$, ES2020) were also measured, following the recommendations of the International Society for the Advancement of Kinanthropometry (Marfell-Jones et al 2006). The body surface area was calculated from the body weight and height measurements, according to DuBois and DuBois (1916). The body density was estimated using the equation of the sum of seven folds (pectoral, subscapular, midaxillary, triceps, suprailiac, abdomen and thigh) developed by Jackson and Pollock (1978), and the Siri equation (Siri 1961) was used to estimate the body fat.

The maximal oxygen uptake $\left(\dot{V} \mathrm{O}_{2 \max }\right)$ was estimated using a submaximal treadmill test according to the recommendations of the ACSM (2010). The exercise protocol consisted of a 3 min warm-up with the treadmill speed $\left(\right.$ Ecafix ${ }^{\circledR}$, EG $700 \times$ ) maintained at $5 \mathrm{~km} \mathrm{~h}^{-1}$. Afterward, this the speed was increased by $1 \mathrm{~km} \mathrm{~h}^{-1}$ every 2 min until reaching $85 \%$ of the heart rate $\left(\mathrm{HR}_{\max }\right)$ previously calculated using the equation proposed by Tanaka et al (2001). The inclination of the treadmill was set at $2 \%$ during the entire test. The cool-down period lasted 2 min with a constant speed of $3 \mathrm{~km} \mathrm{~h}^{-1}$ and a $0 \%$ gradient.

In the methodology proposed by the ACSM (2010), the individual equations for estimating $\dot{V} \mathrm{O}_{2 \max }$ are formulated by linear regression using the values of heart rate (HR) (bpm) and

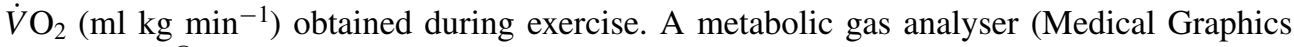
Corporation ${ }^{\circledR}$, VO2000) was used to evaluate the oxygen consumption, a heart monitor (Polar Team2 Pro ${ }^{\circledR}$ ) was used to measure the HR and software (SigmaPlot ${ }^{\circledR}, 12.0$ ) was used to perform the linear regressions.

The subjects were instructed to not perform any type of exercise for $24 \mathrm{~h}$ prior to the day of each experiment.

\section{Procedures for the days of the experiment}

In the morning between 8:00 to 8:30 am, at home, subjects ingested a thermal pill to evaluate their core temperature $\left(T_{\mathrm{C}}\right)$ through a telemetry system (HQ CorTemp ${ }^{\circledR}$ Inc., HT150002). This pill transmits low frequency radio waves with a different wavelength depending on temperature. This radio wave is received and converted into digital format by a data recorder (CorTemp ${ }^{\circledR}$ HQ Inc, HT150016). Each pill was properly calibrated and certified by the manufacturer. All procedures followed the recommendations proposed by Byrne and Lim (2007), which highlight the validity of this type of equipment for recording $T_{\mathrm{C}}$.

From 11:00 to 12:00 h, the subjects had lunch consisting of foods commonly consumed in their daily routine. To avoid physical and thermal stress, the volunteers were transported by car to the laboratory. The volunteers entered the laboratory by 13:30 $\mathrm{h}$ and adapted to room temperature for $1 \mathrm{~h}$. The room had been properly equipped with artificial fluorescent 


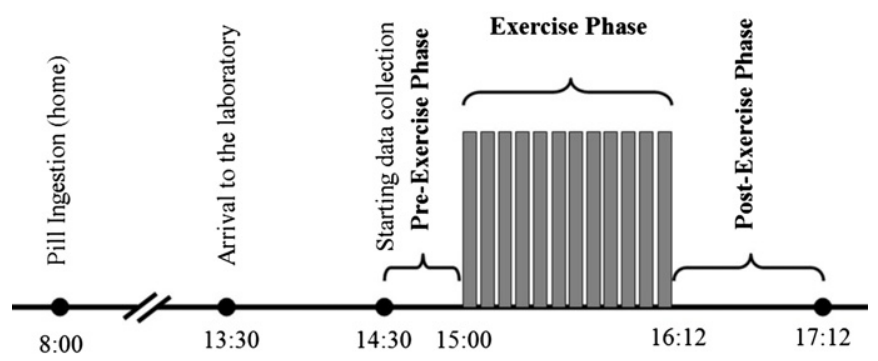

Figure 1. Data collection protocol.

lamps, and the environmental temperature was maintained through a heating/cooling air conditioner (Komeco ${ }^{\circledR}$, Hi-wall Split). The average temperature remained at $24.9 \pm 0.6{ }^{\circ} \mathrm{C}$ with a relative humidity of $62.3 \pm 5.7 \%$ on the IRT experiment days, and the temperature was $24.8 \pm 0.4{ }^{\circ} \mathrm{C}$ with a relative humidity at $61.9 \pm 5.4 \%$ on the thermocouple experiment days. Both measures were recorded using a digital weather station and anemometer (Instrutherm ${ }^{\mathbb{}}$, AD-250), which characterized the environment as temperate (Bain and Jay 2011) with null wind. These environmental conditions are in accordance with the recommendations of Ring and Ammer (2000) for collecting IRT data. HR was monitored at all stages of the study using a heart monitor (Polar Team2 Pro ${ }^{\circledR}$ ). Figure 1 shows the data collection protocol.

The recommendations of the European Association of Thermology (Ring and Ammer 2006) were adopted for $T_{\mathrm{SK}}$ measurements. The main points of these recommendations include that volunteers should avoid the following for $24 \mathrm{~h}$ before the test: caffeine consumption, smoking, alcohol consumption, physical activity, massage and application of any cream, gel or spray in the evaluated body ROI.

During the data collection, subjects wore only training shoes, swimming trunks and the belt of the HR monitor. Immediately upon arrival in the laboratory, each subject drank $500 \mathrm{ml}$ of water to avoid dehydration at the beginning of the exercise.

\section{Pre-exercise phase}

Because body temperature varies with time of day (Heikens et al 2011), the $T_{\mathrm{SK}}$ data collection always began at 14:30. After the adaptation period, the subjects were instructed to indicate their thermal sensation (S) on a nine-point scale and their thermal comfort (C) on seven-point scale; these scales were predetermined in the study of Yasuoka et al (2012).

The equation proposed by Nadel et al (1973) was used to calculate the $M T_{\mathrm{SK}}$ and employs the following eight points: forehead, chest, abdomen, shoulder blade, arm, forearm, thigh and leg. Thus, the following equation was used:

$$
\begin{aligned}
M T_{\mathrm{SK}}=0.21 \times & T_{\text {forehead }}+0.1 \times T_{\text {chest }}+0.17 \times T_{\text {abdomen }}+0.11 \times T_{\text {scapula }}+0.12 \times T_{\text {arm }} \\
& +0.06 \times T_{\text {forearm }}+0.15 \times T_{\text {thigh }}+0.08 \times T_{\text {leg }} .
\end{aligned}
$$

During thermocouple assessments, the subjects remained standing for $30 \mathrm{~min}$ in the test room, and their temperature was recorded every 5 min for seven measurements in a resting condition. The thermocouples were attached to the skin with microporous hypoallergenic tape $\left(\mathrm{Cremer}^{\circledR}\right)$ and were positioned at specific anatomical points according to the instructions provided in the study of Choi et al (1997). Two digital and portable type K/J thermometers, with four channels (Instrutherm ${ }^{\circledR}, \mathrm{TH}-096$ ) and a measurement range from -120 to $+500{ }^{\circ} \mathrm{C}$, accuracy of $0.5 \%$, and sensitivity of $0.1{ }^{\circ} \mathrm{C}$, and eight thermocouples (Instrutherm ${ }^{\circledR}, \mathrm{S}-09 \mathrm{~K}$ ), 
with a measurement range from -70 to $+500{ }^{\circ} \mathrm{C}$ and accuracy of $0.5 \%$, were used for these $T_{\mathrm{SK}}$ recordings.

On the IRT assessment days, the subjects followed the same procedures as above, and every $5 \mathrm{~min}$ one IRT recording was performed under resting conditions. During this period, the volunteer remained in an anatomical position in front of the imager at a distance of $3 \mathrm{~m}$ for the measurement of two thermograms (anterior and posterior regions of the body). An imager (Flir ${ }^{\circledR}$, T420), with a measurement range from -20 to $+120{ }^{\circ} \mathrm{C}, 2 \%$ accuracy, sensitivity $\leqslant 0.05{ }^{\circ} \mathrm{C}$, IR spectral band of 7.5 to 13 microns, refresh rate of $60 \mathrm{~Hz}$, auto-focus and a resolution of $320 \times 240$ pixels, was used to obtain the thermograms.

Furthermore, on the two days of the experiment, $T_{\mathrm{C}}$ and HR were recorded as the average during the last minute of each period. At the 0-5 min and 15-20 min intervals, an oxygen consumption collection was performed using the same equipment that was used to calculate $\dot{V} \mathrm{O}_{2 \max }$ on the two days of the experiment.

The subjects were weighed to determine body mass before and after the experiment, and the urine specific gravity was determined using a refractometer $\left(\mathrm{LF}^{\circledR}, 107 / 3\right)$ from aliquots of urine collected in $50 \mathrm{ml}$ plastic bottles before, immediately after the start activity and post-experiment to check the level of water loss that was induced by the intervention.

\section{Exercise phase}

On the two days of the experiment, the subjects completed an interval test on a treadmill consisting of 12 blocks of $5 \mathrm{~min}$ each separated by an interval of $1 \mathrm{~min}$. The intensity of the blocks of exercise was individually fixed to $60 \%$ of the $\dot{V} \mathrm{O}_{2 \max }$ speed obtained in the pre-session experiment.

Temperature was measured using the thermocouples or IRT during the intervals, and the remaining variables were measured as described for the pre-exercise period. Oxygen consumption was recorded during blocks $1,4,7$ and 10 .

Dehydration can also affect thermoregulatory responses (Gonzalez-Alonso et al 2008, Marins 2011). Therefore, before starting each exercise block, the subjects ingested $1 \mathrm{ml} \mathrm{kg}^{-1}$ of body weight of water to ensure hydration. $T_{\mathrm{C}}$ and HR were recorded for each block of exercise as the average during the last minute of each period.

\section{Post-exercise phase}

The subjects remained standing for $60 \mathrm{~min}$ in the test room, and every $5 \mathrm{~min}$ temperature values were recorded using the thermocouples or IRT for a total of 12 measurements during the entire resting phase. The remaining variables were measured in the same manner as during the pre-exercise period. The oxygen consumption was recorded at the following times: 0-5, 15-20, 30-35 and 45-50 min. During the recovery period, the subjects did not exert any physical effort, did not bathe, did not dry their skin with any type of absorbent material and did not urinate; the only sweat evaporation during this period occurred naturally.

\section{Thermal image processing}

After collecting the IRT, a $1 \mathrm{~cm}^{2}$ area was set up in each reference location (ROI), as shown in figure 2: the forehead, chest, abdomen, shoulder blade, arm, forearm, thigh and leg. Temperatures from each ROI were collected using specific software (Flir Tools ${ }^{\circledR}$ ). The emissivity value adopted for human skin was 0.98 (Lahiri et al 2012), and the reflected room temperature was $23{ }^{\circ} \mathrm{C}$. 


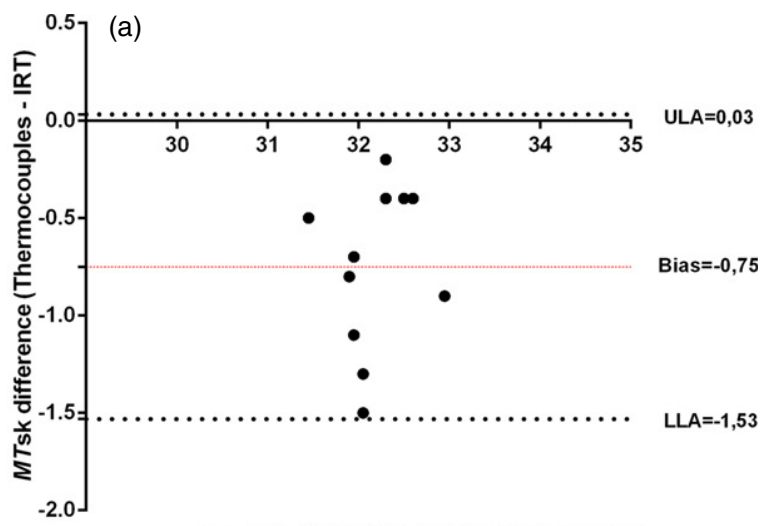

Average MTsk (Thermocouples and IRT)

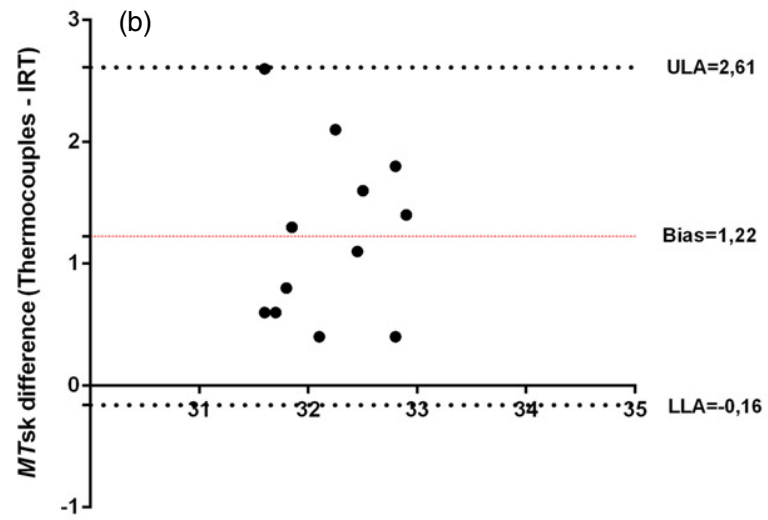

Average MTsk (Thermocouples and IRT)

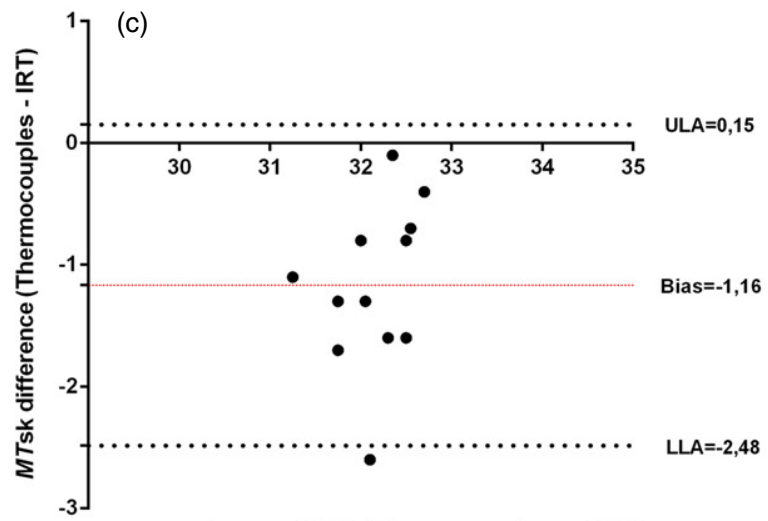

Average $M T$ sk (Thermocouples and IRT)

Figure 2. Bland-Altman plots with upper and lower limits of agreement (ULA and LLA, $95 \%$ ) between the $M T_{\mathrm{SK}}$ values obtained using the thermocouples and IRT. (a) With $15 \mathrm{~min}$ of pre-exercise, (b) $30 \mathrm{~min}$ of exercise and (c) $30 \mathrm{~min}$ post-exercise.

\section{Statistical analysis}

The Shapiro-Wilk test was used to assess the normality of the data. Because the data were normal, descriptive statistics, such as the mean and standard deviation $( \pm \mathrm{SD})$, were subsequently calculated. To compare the $T_{\mathrm{SK}}$ (arm and thigh) obtained using thermocouples 
and IRT at the different time points studied as well as to analyse $T_{\mathrm{C}}, \mathrm{HR}$ and $\dot{V} \mathrm{O}_{2}$, we used the one-way ANOVA for repeated measures, followed by the post-hoc Holm-Sidak test. The paired $t$-test was used to compare variables (body mass, density of urine, $\mathrm{S}$ and $\mathrm{C}$ ) in the pre-and post-exercise conditions and to compare the thermocouple and IRT measurements of $T_{\mathrm{SK}}$ (arm and thigh) and $M T_{\mathrm{SK}}$ at the measured time points.

The limits of agreement between the pairs of $M T_{\mathrm{SK}}$ values obtained using thermocouples and IRT were determined according to the method suggested by Bland and Altman (1986). The coefficient of variation (CV) (Atkinson and Nevill 1998) and the intraclass correlation coefficient (ICC) were also used as measures of the reliability between pairs of $T_{\mathrm{SK}}$ values from the two methods (thermocouples and IRT). The use of these three approaches follows the recommendations about reliability (Atkinson and Nevill 1998) because there are advantages and disadvantages for each approach. The level of significance was $\alpha<0.05$. All analyses were performed using statistical software (Sigmaplot ${ }^{\circledR}, 12.0$ and $\operatorname{SPSS}^{\circledR}, 17.0$ ).

\section{Results}

Table 1 shows the physiological and subjective responses obtained in the study using thermocouples and IRT for the three measurement stages: (a) $15 \mathrm{~min}$ of pre-exercise, (b) $30 \mathrm{~min}$ of exercise and (c) 30 min post-exercise.

The $\mathrm{CV}$ between the methods of measuring $M T_{\mathrm{SK}}$ was $1.7 \%$ in the pre-exercise period, $2.7 \%$ in the exercise period and $2.6 \%$ in post-exercise period. The ICC between the methods of measuring $M T_{\mathrm{SK}}$ was 0.75 [0.12-0.93] in the pre-exercise period, 0.49 [ $\left.-0.80-0.85\right]$ in the exercise period and $0.35[-1.22-0.81]$ post-exercise period, with a confidence interval of $95 \%$ in all of them. The reliability of the $M T_{\mathrm{SK}}$ obtained using the two methods in this study in the pre-, during and post-exercise periods was verified by analysis of the Bland-Altman plot residual scores (figure 2). Even though the number of cases is probably short, these scores show the degree of agreement between the pairs of measurements. These results indicate that the $M T_{\mathrm{SK}}$ obtained from the thermocouples and IRT present heteroscedastic errors, showing the low concordance between the methods.

Figure 3 shows the $M T_{\mathrm{SK}}$ values at different times that were obtained from the thermocouples and IRT. For better visualization, we highlighted the results from the statistical analysis comparing just the $30 \mathrm{~min}$ pre-exercise point (considered the baseline thermal equilibrium) with the other times. In addition, figure 3 summarizes the moment-to-moment $M T_{\mathrm{SK}}$ obtained using the two techniques.

Figure 4 shows the $T_{\mathrm{SK}}$ values for the arm (non-active region) and thigh (active region) at different times before, during and after exercise for the thermocouples and IRT. As in figure 2, we highlighted the statistical analysis results comparing the values at the $30 \mathrm{~min}$ pre-exercise point with those at other times.

\section{Discussion}

The main result of this study was the low concordance found between thermocouples and IRT $M T_{\mathrm{SK}}$ measurements in the pre-exercise, during exercise and post-exercise periods. These results were confirmed by the $\mathrm{CV}$, the ICC and the Bland-Altman plot analyses (figures 2 and 3). This finding agrees with those of Sund-Levander et al (2002) for the $T_{\mathrm{C}}$. They reported that each method obtained different responses with different normal ranges. Thus, the present study confirms that comparing the results from IRT with those from thermocouples would not be ideal in all situations studied for this work because the $M T_{\mathrm{SK}}$ measured using thermocouples 
Table 1. Physiological and subjective responses obtained using thermocouples and IRT in the pre-exercise (Pre-ex), exercise and post-exercise (Post-ex) states.

\begin{tabular}{|c|c|c|c|c|c|c|}
\hline & \multicolumn{3}{|c|}{ IRT } & \multicolumn{3}{|c|}{ Thermocouples } \\
\hline & Pre-ex & Exercise & Post-ex & Pre-ex & Exercise & Post-ex \\
\hline Body mass (kg) & $73.8 \pm 6.3$ & & $73.3 \pm 6.3^{\mathrm{a}}$ & $73.5 \pm 6.4$ & & $73.2 \pm 6.4^{\mathrm{a}}$ \\
\hline Urine density (sg) & $1014.1 \pm 5.6$ & & $1013.1 \pm 5.8$ & $1013.9 \pm 4.5$ & & $1013.7 \pm 4.9$ \\
\hline $\mathrm{S}$ (points) & $-0.5 \pm 0.9$ & & $0.2 \pm 0.9$ & $-0.3 \pm 1$ & & $-0.2 \pm 0.9$ \\
\hline $\mathrm{C}$ (points) & $1.5 \pm 0.9$ & & $1.9 \pm 1.2$ & $1.2 \pm 1$ & & $1.6 \pm 1.1$ \\
\hline$\dot{V} \mathrm{O}_{2 \max }\left(\mathrm{ml} \mathrm{kg} \mathrm{min}^{-1}\right)$ & $5.2 \pm 0.8$ & $26.5 \pm 2.3^{\mathrm{b}}$ & $6.1 \pm 1.6^{\mathrm{c}}$ & $5.1 \pm 0.6$ & $26.1 \pm 3.9^{\mathrm{b}}$ & $6.0 \pm 1.1^{\mathrm{c}}$ \\
\hline $\mathrm{HR}(\mathrm{bpm})$ & $71.1 \pm 12.8$ & $137.6 \pm 16.6^{\mathrm{d}}$ & $90.9 \pm 12.6^{\mathrm{d}}$ & $72.3 \pm 13.9$ & $136.9 \pm 15.8^{\mathrm{d}}$ & $92.1 \pm 14.4^{\mathrm{d}}$ \\
\hline$T_{\mathrm{C}}\left({ }^{\circ} \mathrm{C}\right)$ & $37.2 \pm 0.3$ & $37.8 \pm 0.4^{\mathrm{d}}$ & $37.4 \pm 0.3$ & $37.2 \pm 0.2$ & $37.7 \pm 0.3^{\mathrm{d}}$ & $37.3 \pm 0.2$ \\
\hline
\end{tabular}

Pre-ex $=$ pre-exercise; Post-ex = post-exercise; $S=$ thermal sensation; $C=$ thermal comfort $=\mathrm{CT} ; T_{\mathrm{C}}=$ core temperature.

${ }^{a}$ Significant differences $p<0.001$ between pre-ex versus post-ex in same condition.

b Significant differences $p<0.001$ between pre-ex versus exercise in same condition.

${ }^{\mathrm{c}}$ Significant differences $p<0.001$ between exercise versus post-ex in same condition.

${ }^{\mathrm{d}}$ Significant differences $p<0.001$ between this point versus others in same condition. 


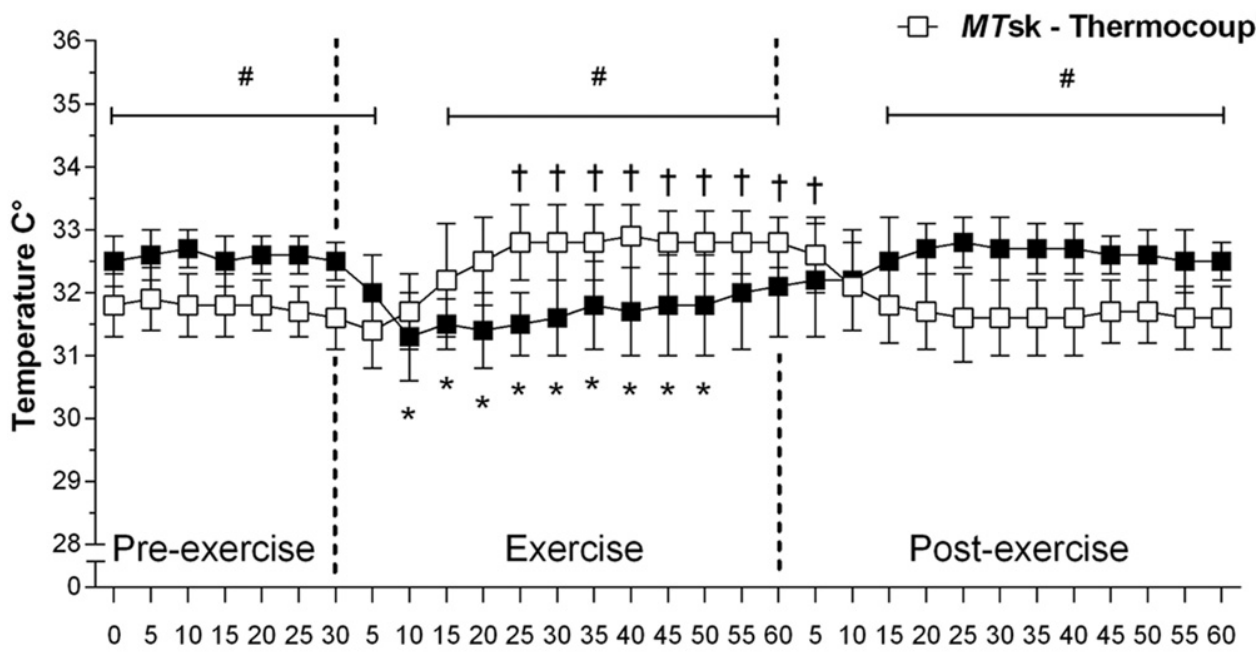

\section{Time (min)}

Figure 3. $M T_{\mathrm{SK}}$ values at different times pre-, during and post-exercise obtained from thermocouples and IRT. The results are presented as the mean \pm SD. * Significant differences between minute 30 pre-exercise versus post-exercise for IRT. 'Significant differences between minute 30 pre-exercise versus post-exercise for thermocouples. \#Significant differences between IRT and thermocouple results.

is higher than that measured using IRT during exercise, and lower in the pre- and post-exercise conditions (figure 3 ).

The pre-, during and post-exercise results indicate that the physiological responses of the subjects were similar when IRT and thermocouples were used. We think that the adopted hydration procedure maintained the hydration state at the end of the experiments. Although it was considered statistically significant in both experiments, the highest water loss was only $0.7 \%$ of the body mass for IRT and $0.5 \%$ for the thermocouples. There were no changes in the density of the urine, clearly indicating again that the hydration of the subjects was maintained (Casa et al 2000). The increase in $T_{\mathrm{C}}$, HR and oxygen consumption was sufficient to start the thermoregulatory responses during exercise, such as sweating and blood flow redistribution, that are expected for the intense physical stimulus generated (Gonzalez-Alonso et al 2008).

In the pre-exercise condition, the statistical analyses indicate poor agreement between the $M T_{\mathrm{SK}}$ values from the two methods. Our results indicated that the correlation between the thermocouples and IRT showed an averaged error of $-0.75{ }^{\circ} \mathrm{C}$ with a confidence interval of $95 \%$ ranging from 0.03 to $-1.52{ }^{\circ} \mathrm{C}$ (figure 2 ). In contrast, in the study of van den Heuvel et al (2003), which compared thermocouple and IRT temperatures during sleep, the average error was $2.30{ }^{\circ} \mathrm{C}$ with a confidence interval of $95 \%$ ranging from 1.25 to $3.39{ }^{\circ} \mathrm{C}$; this result reinforces the low correlation between the values of $T_{\mathrm{SK}}$ measured by these methods. Importantly, there was disagreement between the findings of the study of van den Heuvel et al (2003) and those presented in this study, where the values of $T_{\mathrm{SK}}$ obtained by IRT are higher than those recorded by the thermocouples. A possible explanation for these differences is the type of equipment employed; the substances and the characteristics of the sticker for attaching the thermocouple may lead to different temperature measurements (Tyler 2011).

It is also possible to speculate that the higher $M T_{\mathrm{SK}}$ pre-exercise values recorded by IRT are partly explained by the physical principles that guide these techniques. Thermocouples 


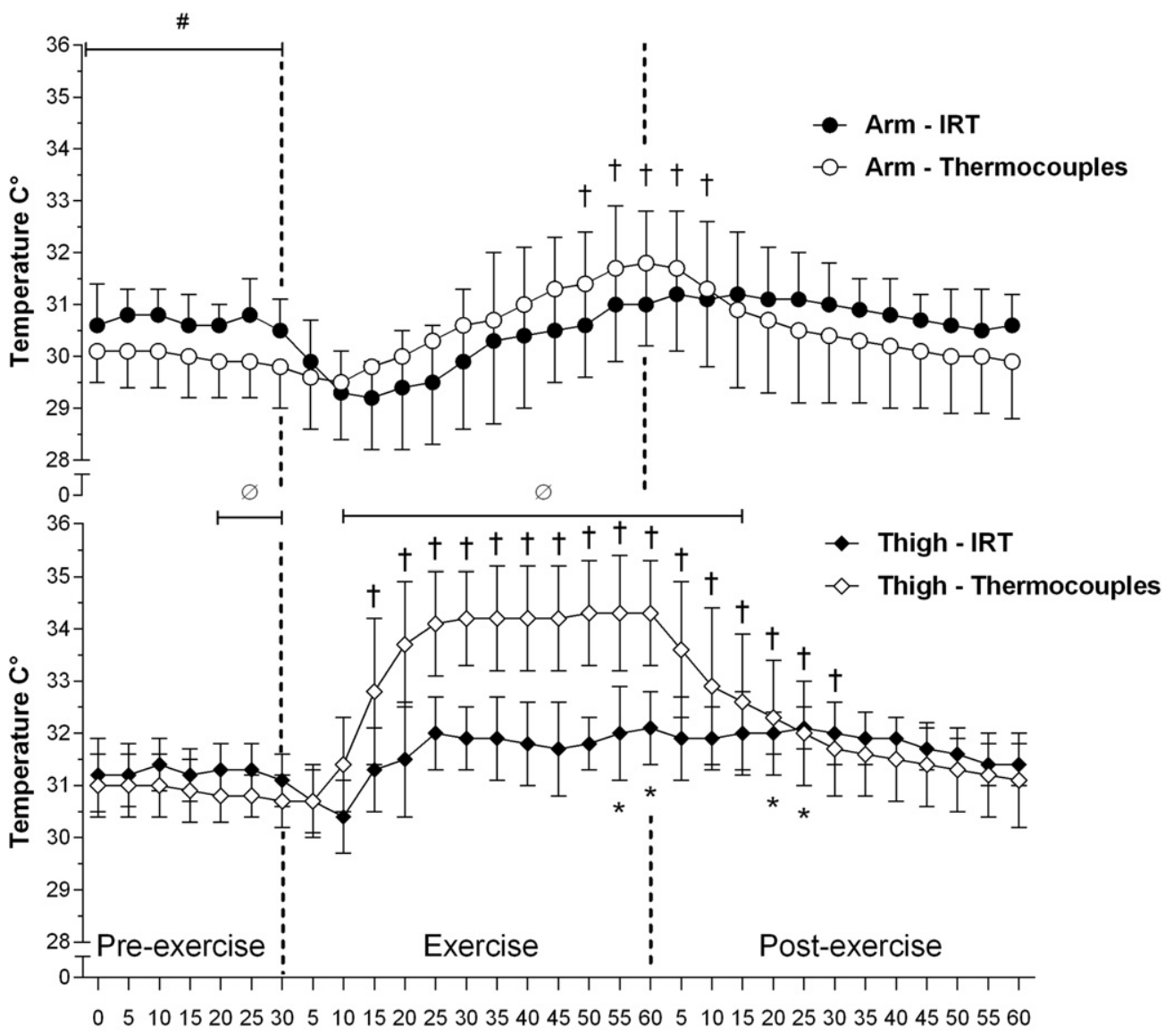

Time (min)

Figure 4. $T_{\mathrm{SK}}$ values for the pre-exercise, exercise and post-exercise periods obtained using thermocouples and IRT in the arm and thigh regions. The results are presented as the mean \pm SD. ${ }^{*}$ Significant differences between minute 30 pre-exercise versus later moments of the protocol measured by IRT. 'Significant differences between minute 30 pre-exercise versus later moments of the protocol measured by thermocouples. ${ }^{\#}$ Significant differences between IRT and thermocouples in the arm. ${ }^{\varnothing}$ Significant differences between IRT and thermocouples in the thigh.

transform the voltage generated by a conductor subjected to a thermal gradient into temperature values, while IRT changes the energy emitted by radiation to temperature values. At rest, most (approximately 60\%) of the heat loss occurs through radiation (Wendt et al 2007). Therefore, it is possible that the IRT equipment records higher values in that condition.

When we compare the $M T_{\mathrm{SK}}$ results obtained with the thermocouples at $15 \mathrm{~min}$ in the pre-exercise condition $\left(31.8 \pm 0.5^{\circ} \mathrm{C}\right)$ with those of other studies, similar $M T_{\mathrm{SK}}$ values of $31.8 \pm 0.3{ }^{\circ} \mathrm{C}$ (Burdon et al 2010), $31.7 \pm 0.3{ }^{\circ} \mathrm{C}$ (Bain and Jay 2011), and $31.8 \pm$ $0.7{ }^{\circ} \mathrm{C}$ (Bain et al 2011) are found in studies with similar environmental conditions. These results indicate that the measured $M T_{\mathrm{SK}}$ values can be considered normal for a thermocouple evaluation.

Equations are rarely used to calculate the $M T_{\mathrm{SK}}$ in studies using IRT as a measurement technique, making comparisons with other methods difficult. However, the work of Costa 
(2012) presented $M T_{\mathrm{SK}}$ data in the resting condition using IRT $\left(32.2 \pm 0.5^{\circ} \mathrm{C}\right)$, and those data were similar to the mean values $\left(32.5 \pm 0.4{ }^{\circ} \mathrm{C}\right)$ obtained in this study at the $15 \mathrm{~min}$ point. Therefore, these values can be considered normal for an IRT evaluation.

Consistent differences were found between the $M T_{\mathrm{SK}}$ values recorded using the two methods (thermocouples versus IRT) in the resting condition. Significant differences $(p<0.05)$ were measured for the 30 min of continuous monitoring, with the smallest difference of $0.7^{\circ} \mathrm{C}$ at $0 \mathrm{~min}$ and the greatest difference of $0.9^{\circ} \mathrm{C}$ at $25 \mathrm{~min}$ (figure 3). Similar behaviour was observed when analysing specific regions, such as the upper arm and the thigh, in which the $T_{\mathrm{SK}}$ values at rest from IRT were always higher than those from the thermocouples.

During exercise, the $M T_{\mathrm{SK}}$ values already showed a pattern in which higher values were obtained using thermocouples than those from IRT; this difference was opposite to that observed at rest. The agreement between the thermocouples and IRT showed an average error $1.22{ }^{\circ} \mathrm{C}$ with a confidence interval of $95 \%$ ranging from 2.61 to $-0.16{ }^{\circ} \mathrm{C}$; thus, the average error increased with exercise performance (figure 2(b)).

An important $M T_{\mathrm{SK}}$ response observed during the exercise condition was that the temperature measured using the thermocouples increased compared with that measured during the resting condition. In contrast, the $M T_{\mathrm{SK}}$ measured using IRT during exercised decreased compared with that measured at rest for both the general and local analyses (as shown in figure 4). During the exercise phase, the $M T_{\mathrm{SK}}$ obtained by the thermocouples exceeded that from the IRT after minute 15 , with the largest difference of $1.25{ }^{\circ} \mathrm{C}$ occurring at minute 25 (figure 3). Analyses of the arm (non-active muscles) temperatures indicate that there was a significant difference $p<0.001$ between the $T_{\mathrm{SK}}$ obtained by the thermocouples and those from the IRT during exercise (figure 4); however, the thigh ROI $T_{\mathrm{SK}}$ (active muscles) shows significant differences $p<0.001$ beginning at $10 \mathrm{~min}$ of exercise with the highest difference of $1.25{ }^{\circ} \mathrm{C}$ occurring at $25 \mathrm{~min}$ of exercise (figure 4). These findings clearly indicate that both measurement techniques can lead to different results considering active and non-active areas during exercise; so that, the analysis of data and the comparison of studies carried out with both methods should be done carefully.

Several factors can explain the observed differences between the data obtained by both methods during exercise: the pressure exerted by fixation methods (Tyler 2011), the use of substances for the fixation (Tyler 2011), and the heat loss through convection and evaporation in the region where thermocouple was fixed (Tyler 2011, Buono et al 2007), which may cause the $T_{\mathrm{SK}}$ measured by the thermocouples to increase. Furthermore, for IRT, there is an easier loss of heat by radiation, convection and evaporation during exercise mainly due to higher sweat production, which may result in a lower $T_{\mathrm{SK}}$ under these conditions. The study by Clark et al (1977) reported a difference in the $T_{\mathrm{SK}}$ values measured by thermocouples and IRT $\left( \pm 1.5^{\circ} \mathrm{C}\right)$, although differences were reported to reach $4.0^{\circ} \mathrm{C}$. Another factor that may have contributed to the divergent results during exercise could be that the use of IRT allows greater heat loss by convection and evaporation and a consequently can record lower $T_{\mathrm{SK}}$ for the ROI.

Analysing figure 4 indicates that the $T_{\mathrm{SK}}$ values recorded in the arms (non-active region) are lower than those recorded in the active region, i.e., the region with the highest concentration of blood flow and higher production heat by the active muscles. In addition to the particularities of each method, this difference effectively contributes to these results.

The $M T_{\mathrm{SK}}$ values obtained using thermocouples after $60 \mathrm{~min}$ of exercise were $32.8 \pm$ $0.4{ }^{\circ} \mathrm{C}$, which are similar to those obtained by Burdon et al $(2010)\left(32.9 \pm 0.4{ }^{\circ} \mathrm{C}\right)$ or slightly lower $\left(33.1 \pm 0.9^{\circ} \mathrm{C}\right)$ than Bain et al $(2011)$. Thus, the results of this study indicate that the $M T_{\mathrm{SK}}$ values can be considered normal for an evaluation with thermocouples. Reductions in $T_{\mathrm{SK}}$ for certain ROIs during exercise have been reported in different studies (Merla et al 2010, Akimov et al 2010, Zontak et al 1998, Torii et al 1992). However, no studies have identified the 
equations used to calculate the $M T_{\mathrm{SK}}$ while using IRT as a measurement technique, preventing a comparison with our data. Thus, the values found in this study can be considered a reference for the $M T_{\mathrm{SK}}$ behaviour measured using IRT in future work.

The statistical analyses for the return to the resting condition also indicate a poor correlation between the values obtained using the two methods for assessing $M T_{\mathrm{SK}}$. The $M T_{\mathrm{SK}}$ is even greater at rest, with a mean error of $-1.16^{\circ} \mathrm{C}$ and a confidence interval of $95 \%$ ranging from 0.15 to $-2.48{ }^{\circ} \mathrm{C}$ (figure 2(c)). At this point, the $M T_{\mathrm{SK}}$ measured using IRT is higher than that measured using thermocouples. The physical principles of each technique may be crucial to these results.

After 15 min of recovery, the $M T_{\mathrm{SK}}$ trend inverts between the two methods. At this moment, the $M T_{\mathrm{SK}}$ measured using IRT increases, and the $M T_{\mathrm{SK}}$ values measured using thermocouples decrease; this situation is similar to that at rest in the pre-exercise condition (figure 3 ).

The difference between the $M T_{\mathrm{SK}}$ values recorded using the two methods (thermocouples versus IRT) during the post-exercise condition is smallest $\left(0.1^{\circ} \mathrm{C}\right)$ at $10 \mathrm{~min}$ and highest $\left(1.2{ }^{\circ} \mathrm{C}\right.$ ) at $25 \mathrm{~min}$ (figure 3). For the specific regions of the arm and thigh, the $T_{\mathrm{SK}}$ values measured using IRT were always higher, but not to a statistically significant extent, than those recorded using thermocouples 25 min after finishing the exercise.

The limitations of this study include the fact that sweating was not quantified and the headwind action for facilitating heat loss by convection on the treadmill was not simulated. It may also be considered that the movement of the surface attached to the thermocouples during exercise (stretches) might have contributed to increasing the value of the data obtained by them.

We think that the results of this study could add important information for understanding of the thermoregulatory adjustments produced during exercise. The results obtained in this study may be different in other conditions: exercise performed at different intensities; exercise in other modalities, such as cycling; exercise in an external environment; exercise under different weather conditions; or even exercise performed by female or obese subjects.

\section{Conclusion}

There is low agreement between the $M T_{\mathrm{SK}}$ values measured using thermocouples and IRT for pre-, during and post-exercise conditions, demonstrating the weak reliability between the two forms of measurement. Comparing the results of studies conducted using both methods is not appropriate in all the conditions studied in this work. Moreover, the use of thermocouples showed higher $M T_{\mathrm{SK}}$ values during exercise compared with those measured in the preexercise condition. In contrast, IRT data showed a reduction in the $M T_{\mathrm{SK}}$, indicating that the interpretation will vary depending on the method employed.

\section{Acknowledgments}

We acknowledge CAPES for the Masters Scholarship and FAPEMIG for funding project number: 00356-11.

\section{References}

Ahmadi N, McQuilkin G L, Akhtar M W, Hajsadeghi F, Kleis S J, Hecht H, Naghavi M and Budoff M 2011 Reproducibility and variability of digital thermal monitoring of vascular reactivity Clin. Physiol. Funct. Imaging 31 422-8 
Akimov E, Andreev R, Kalenov Y, Kirdin A, Son'kin V and Tonevitsky A 2010 The human thermal portrait and its relations with aerobic working capacity and the blood lactate level Hum. Physiol. 36 447-56

American College of Sports Medicine (ACSM) Thompson W R, Gordon N F and Pescatello L S 2010 ACSM's Guidelines for Exercise Testing and Prescription (Philadelphia, PA: Lippincott)

Atkinson G and Nevill A M 1998 Statistical methods for assessing measurement error (reliability) in variables relevant to sports medicine Sports Med. 26 217-38

Bain A and Jay O 2011 Does summer in a humid continental climate elicit an acclimatization of human thermoregulatory responses Eur. J. Appl. Physiol. 111 1197-205

Bain A R, Deren T M and Jay O 2011 Describing individual variation in local sweating during exercise in a temperate environment Eur. J. Appl. Physiol. 111 1599-607

Bland J M and Altman D G 1986 Statistical methods for assessing agreement between two methods of clinical measurement Lancet $1307-10$

Bouzida N, Bendada A and Maldague X P 2009 Visualization of body thermoregulation by infrared imaging J. Therm. Biol. 34 120-6

Buono M J, Jechort A, Marques R, Smith C and Welch J 2007 Comparison of infrared versus contact thermometry for measuring skin temperature during exercise in the heat Physiol. Meas. 28855

Burdon C, O’Connor H, Gifford J, Shirreffs S, Chapman P and Johnson N 2010 Effect of drink temperature on core temperature and endurance cycling performance in warm, humid conditions J. Sports Sci. 28 1147-56

Byrne C and Lim C L 2007 The ingestible telemetric body core temperature sensor: a review of validity and exercise applications Br. J. Sports Med. 41 126-33

Campbell I 2011 Body temperature and its regulation Anaesth. Intensive Care Med. 12 240-4

Casa D J, Armstrong L E, Hillman S K, Montain S J, Reiff R V, Rich B S, Roberts W O and Stone J A 2000 National athletic trainers' association position statement: fluid replacement for athletes J. Athl. Train. 35 212-24

Charkoudian N 2010 Mechanisms and modifiers of reflex induced cutaneous vasodilation and vasoconstriction in humans J. Appl. Physiol. 109 1221-8

Childs P R N 2001 Thermocouples Practical Temperature Measurement (Oxford: ButterworthHeinemann) pp 98-144

Chisholm D M, Collis M L, Kulak L L, Davenport W and Gruber N 1975 Physical activity readiness Br. Col. Med. J. 17 375-8

Choi J K, Miki K, Sagawa S and Shiraki K 1997 Evaluation of mean skin temperature formulas by infrared thermography Int. J. Biometeorol. 41 68-75

Clark R P, Mullan B J and Pugh L G 1977 Skin temperature during running — a study using infra-red colour thermography J. Physiol. 267 53-62

Costa C M A 2012 Técnicas de mensuração da temperatura corporal: uma especial atenção para as variações da temperatura da pele mensuradas por termografia ao longo do dia (Viçosa: Universidade Federal de Viçosa)

Davie A and Amoore J 2010 Best practice in the measurement of body temperature Nurs. Stand. 24 42-9

Du Bois D and DuBois E F 1916 A formula to estimate the approximate surface area if height and weight be known Arch. Intern. Med. 17 863-71

Fernandes A A, Amorim P R S, Prímola-Gomes T N, Sillero-Quintana M, Fernández Cuevas I, Silva R G, Pereira J C and Marins J C B 2012 Avaliação da temperatura da pele durante o exercício através da termografia infravermelha: uma revisão sistemática Rev. Andal. Med. Deporte 5 113-7

Garber C E, Blissmer B, Deschenes M R, Franklin B A, Lamonte M J, Lee I M, Nieman D C and Swain D P 2011 American College of Sports Medicine position stand. Quantity and quality of exercise for developing and maintaining cardiorespiratory, musculoskeletal, and neuromotor fitness in apparently healthy adults: guidance for prescribing exercise Med. Sci. Sports Exerc. 43 1334-59

Gonzalez-Alonso J, Crandall C G and Johnson J M 2008 The cardiovascular challenge of exercising in the heat J. Physiol. 586 45-53

Hasselberg M J, McMahon J and Parker K 2011 The validity, reliability, and utility of the iButton ${ }^{\circledR}$ for measurement of body temperature circadian rhythms in sleep/wake research Sleep Med. $145-11$

Heikens M J, Gorbach A M, Eden H S, Savastano D M, Chen K Y, Skarulis M C and Yanovski J A 2011 Core body temperature in obesity Am. J. Clin. Nutr. 93 963-7 
Jackson A S and Pollock M L 1978 Generalized equations for predicting body density of men $B r . J$. Nutr. 40 497-504

Johnson J M 2010 Exercise in a hot environment: the skin circulation Scand. J. Med. Sci. Sports 20 (Suppl. 3) 29-39

Johnson J M and Kellogg D L Jr 2010 Thermoregulatory and thermal control in the human cutaneous circulation Front. Biosci. 2 825-53

Jones B F 1998 A reappraisal of the use of infrared thermal image analysis in medicine IEEE Trans. Med. Imaging 17 1019-27

Kennedy D A, Lee T and Seely D 2009 A comparative review of thermography as a breast cancer screening technique Integr. Cancer Ther. 8 9-16

Lahiri B B, Bagavathiappan S, Jayakumar T and Philip J 2012 Medical applications of infrared thermography: a review Infrared Phys. Technol. 55 221-35

Marfell-Jones M, Olds T and Stewart A L C 2006 International Standards for Anthropometric Assessment (Potchefstroom: ISAK)

Marins J C B 2011 Hidratação na atividade física e no esporte: equilíbrio hidromineral (Jundiaí: Fontoura)

McArdle W, Katch F and Katch V 2001 Fisiologia do Exercício: Nutrição e Desempenho Humano (Rio de Janeiro: Guanabara Koogan)

McCoy M, Campbell I, Stone P, Fedorchuk C, Wijayawardana S and Easley K 2011 Intra-examiner and inter-examiner reproducibility of paraspinal thermography PloS one 6 e16535

Merla A, Mattei P A, Di Donato L and Romani G L 2010 Thermal imaging of cutaneous temperature modifications in runners during graded exercise Ann. Biomed. Eng. 38 158-63

Nadel E R, Mitchell J W and Stolwijk J A J 1973 Differential thermal sensitivity in the human skin Pflugers Arch. Eur. J. Phys. 340 71-6

Ring E and Ammer A 2000 The technique of infra red imaging in medicine Thermology Int. 10 7-14

Ring E F and Ammer K 2006 Standard procedures for infrared imaging in medicine Medical Devices and Systems. Electrical Engineering Handbook (Boca Raton, FL: CRC Press) pp 31-6

Ring E F J and Ammer K 2012 Infrared thermal imaging in medicine Physiol. Meas. 33 R33

Shibasaki M, Wilson T E and Crandall C G 2006 Neural control and mechanisms of eccrine sweating during heat stress and exercise J. Appl. Physiol. 100 1692-701

Siri W E 1961 Body composition from fluid spaces and density: analyses of methods Techniques for Measuring Body Composition ed J Brozek and A Henschel (Washington DC: National Academy of Science)

Smith A D H, Crabtree D R, Bilzon J L J and Walsh N P 2010 The validity of wireless iButtons ${ }^{\circledR}$ and thermistors for human skin temperature measurement Physiol. Meas. 3195

Sund-Levander M, Forsberg C and Wahren L K 2002 Normal oral, rectal, tympanic and axillary body temperature in adult men and women: a systematic literature review Scand. J. Caring Sci. 16 122-8

Tanaka H, Monahan K D and Seals D R 2001 Age-predicted maximal heart rate revisited J. Am. College Cardiol. 37 153-6

Torii M, Yamasaki M, Sasaki T and Nakayama H 1992 Fall in skin temperature of exercising man $B r . J$. Sports Med. 26 29-32

Tyler C J 2011 The effect of skin thermistor fixation method on weighted mean skin temperature Physiol. Meas. 321541

van den Heuvel C J, Ferguson S A, Dawson D and Gilbert S S 2003 Comparison of digital infrared thermal imaging (DITI) with contact thermometry: pilot data from a sleep research laboratory Physiol. Meas. 24717

Vargas J V C, Brioschi M L, Dias F G, Parolin M B, Mulinari-Brenner F A, Ordonez J C and Colman D 2009 Normalized methodology for medical infrared imaging Infrared Phys. Technol. 52 42-7

Wendt D, van Loon L J and Lichtenbelt W D 2007 Thermoregulation during exercise in the heat: strategies for maintaining health and performance Sports Med. 37 669-82

Yasuoka A, Kubo H, Tsuzuki K and Isoda N 2012 Interindividual differences in thermal comfort and the responses to skin cooling in young women J. Therm. Biol. 37 65-71

Zaproudina N, Varmavuo V, Airaksinen O and Närhi M 2008 Reproducibility of infrared thermography measurements in healthy individuals Physiol. Meas. 29515

Zontak A, Sideman S, Verbitsky O and Beyar R 1998 Dynamic thermography: analysis of hand temperature during exercise Ann. Biomed. Eng. 26 988-93 